\title{
COMMENT
}

CONSERVATION Comparing tWo calls to embrace humans' impact on nature $\mathbf{p . 2 9}$

\section{COMICS Richard}

Feynman in graphic novel form p.32
PoLICY Call to save Turkish science institute from closure p.33
OBITUARY Baruj Benacerraf, immunology Nobellist, remembered $\mathbf{p . 3 4}$

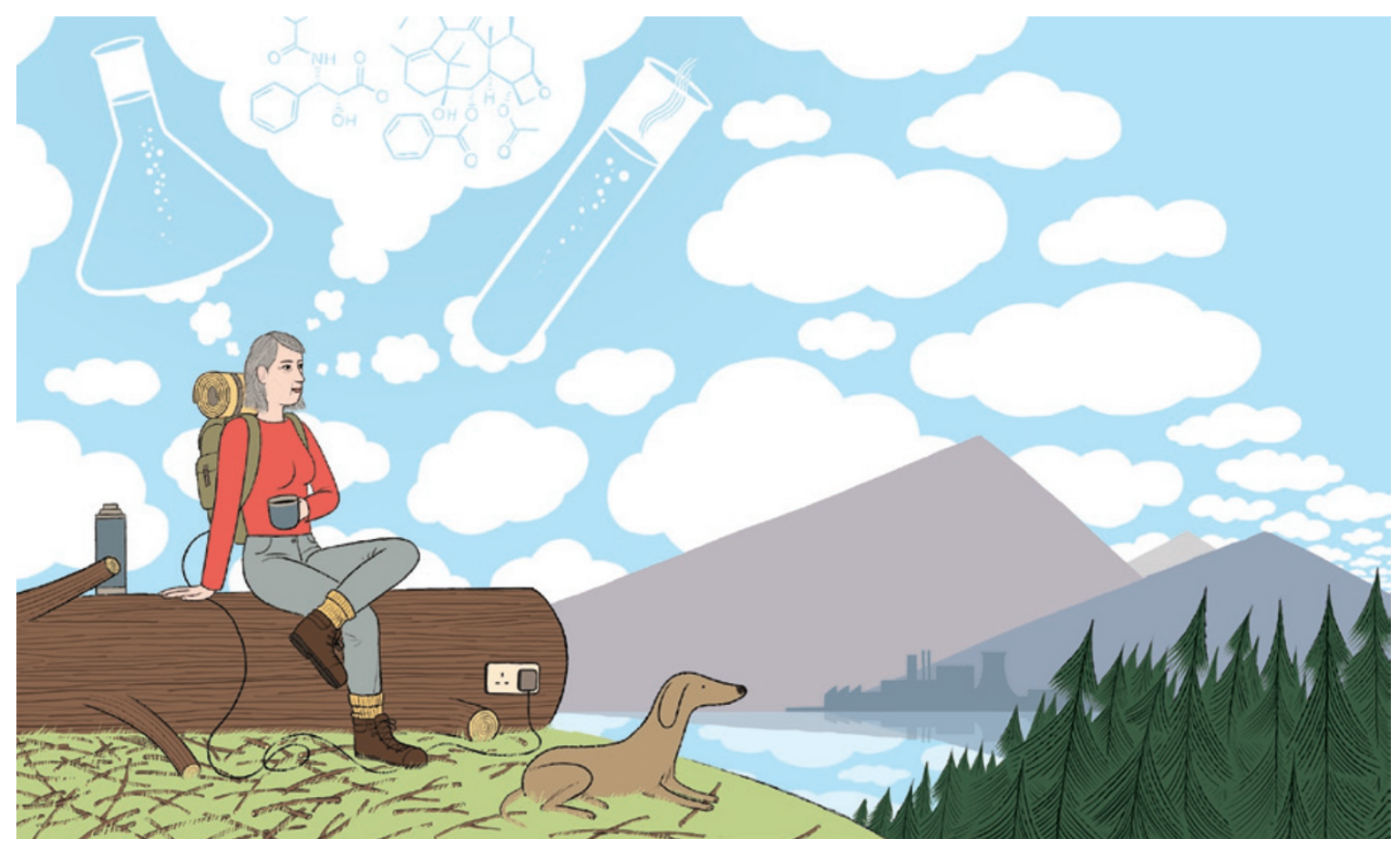

\section{A healthy work-life balance can enhance research}

\section{Scientists should make time for play to complement their intense work, maintain creativity and keep the ideas flowing, argues Julie Overbaugh.}

S triking a balance between work, family, friends and leisure is often hard in science. But there must be room for those who want this balance, otherwise creative people with the potential to make significant contributions to scientific discovery will be excluded.

In my college years at the University of Connecticut in Storrs, I juggled a chemistry major with playing on First Division college basketball and tennis teams. And, ingrained from my Irish upbringing, I felt that college was also meant to be a time where some evenings were spent in the pub with friends. I brought art classes into the mix in graduate school. From these experiences, I learned to value efficiency, balance and teamwork, all of which have influenced my approach to running my research programme. Today I direct a US lab of about 15 people and share responsibility for a much larger international team focused on HIV prevention research.

During my training, I experienced many different lab styles and, in the process, realized that I was not cut out for clocking long hours for the rest of my life. I decided to try to do science in a manner that I could integrate into my life, leaving time for an extensive network of friends and family and other interests, and accept that there would be other paths if this one did not work - a crucial mindset for not succumbing to a frenzied work life.

Twenty years on, I have found that spending long hours in the lab or at the computer does not necessarily promote the creative thinking that is integral to scientific discovery. In fact, I have many of my best ideas while walking the dogs in the morning, riding my bike home from work or weekending in the mountains.

\section{QUALITY NOT QUANTITY}

I have no objection to people who choose to do long hours in the lab, but I have also never expected lab members to give up other 
aspects of their lives to pursue science. One of my team recently noted: "You expect high-quality work - but you do not measure someone's efforts by time spent in the lab in the evenings or on weekends, rather you measure them by the quality of their data."

To be a successful scientist there are times when it is important to pull out all the stops - when a big grant deadline is looming or a high-impact paper is wrapping up. Sometimes, when we are competing with other labs on an exciting story, I briefly imagine locking everyone in the lab to try to push for results more quickly.

By resisting the temptation to drive my group so hard, we might have ended up with the second or third paper on a topic in a less prestigious journal on occasion. But our contributions are clearly recognized. For example: I have given plenary talks at every major HIV meeting; we have received two National Institutes of Health (NIH) MERIT awards; former trainees are faculty members at Harvard University in Cambridge, Massachusetts, Stanford University in California, Baylor College of Medicine in Houston, Texas, and many other top research institutions; others are successful government scientists at the NIH and the Centers for Disease Control and Prevention.

More importantly, in many cases, we solved a problem more effectively, and thus gained some advantage, because people in the lab were less stressed by long hours, constant demands and excessive expectations. In my view, an unremitting pace with no time to step back leads, over the long term, to a fatigued and unhappy team that is not operating at its best.

\section{TIME FOR TEA}

For the past two decades, I have worked in a highly productive interdisciplinary and international collaboration with scientists from many cultures. We are committed to reducing the burden of HIV, particularly in developing countries, and many on the team regularly see the devastation of HIV in clinical practice.

But colleagues also have a range of demands on their time, including young faculty members who juggle research with clinical work and raising children or helping ageing parents. The original collaboration was driven by the needs of the science; it is sustained by esprit de corps. The glue is our appreciation of the specific needs and lifestyle choices of each group member, including our trainees.

I am also fortunate to work at an institution where the focus is on our contributions, not our hours. When a colleague recently suffered a devastating accident, many faculty members and staff were regularly by her hospital bed for months, placing their presence there at higher priority than their work. Thus, it is noteworthy that, among 209 faculty members, we have three Nobel laureates and numerous others who have received prestigious awards (go.nature.com/zebxjt).

Balancing work and other aspects of life is becoming harder in science, as in other professions. We are expected to be constantly responsive to e-mail, alert to rapid online publications, to manage increasing administrative and regulatory demands, and to devote more and more time to securing funding.

Tighter funding and ease of travel have made 'being on tour' an integral part of being the lab chief or principal investigator (PI), often to the exclusion of spending time with the team. Being a lab head can be more PR than PI, with the pressure to 'sell your goods' on the road. I realized that attending just a few meetings a year was enough when I reached the point that I had heard my colleagues speak so many times that I could have given some of their talks myself, and they mine.

Meetings are wonderful for networking and exchanging new findings and ideas, but they can have diminishing returns in terms of promoting creativity or seeding a breakthrough. We should re-evaluate the expanding repertoire of conferences in some fields to determine if the scientific exchange genuinely offsets the demands of constant travel.

More generally, time to think deeply about scientific problems is becoming increasingly rare. Indeed, with the rapid advance of new technologies, many studies simply apply new methods to old problems, often getting a more refined, but similar answer. Although it is comforting to see the consistency in conclusions, the true advances in such cases may be small.

About 25 years ago, I was the lone trainee in a lab of a prominent senior scientist. We spent many afternoons drinking tea and discussing diverse scientific issues. Current scientific discovery would benefit from reinstating this mostly bygone tradition. Imagine, further, if labs everywhere shut down all experiments for a week each year to have everyone read recent - and old literature and discuss ideas over a favourite beverage. I predict that new ideas would emerge and people would be energized.

It is a privilege to have work that is challenging and satisfying, and that contributes to society and helps advance knowledge and training. For some, a total focus on research and the thrill of discovery is fulfilling. For others, originality, productivity and overall happiness in life demands a different mix. There must be room for both types of people in science, as each has the potential to make important contributions. SEE EDITORIAL P.5 AND NEWS FEATURE P.2O

Julie Overbaugh is in the Division of Human Biology, Fred Hutchinson Cancer Research Center, Seattle, Washington 98109, USA. e-mail: joverbau@fhcrc.org 